\title{
$4 f$ excitations in Ce Kondo lattices studied by resonant inelastic x-ray scattering
}

\author{
A. Amorese, ${ }^{1}$ G. Dellea, ${ }^{2}$ M. Fanciulli, ${ }^{1,2}$ S. Seiro, ${ }^{3}$ C. Geibel, ${ }^{3}$ C. Krellner,,${ }^{3,4}$ I. P. Makarova, ${ }^{5}$ L. Braicovich, ${ }^{2}$ \\ G. Ghiringhelli, ${ }^{2}$ D. V. Vyalikh, ${ }^{6,7}$ N. B. Brookes, ${ }^{1}$ and K. Kummer ${ }^{1, *}$ \\ ${ }^{1}$ European Synchrotron Radiation Facility, 71 Avenue des Martyrs, CS40220, F-38043 Grenoble Cedex 9, France \\ ${ }^{2}$ CNR-SPIN and Dipartimento di Fisica, Politecnico di Milano, \\ Piazza Leonardo da Vinci 32, Milano I-20133, Italy \\ ${ }^{3}$ Max Planck Institute for Chemical Physics of Solids, Nöthnitzer Strasse 40, D-01187 Dresden, Germany \\ ${ }^{4}$ Kristall- und Materiallabor, Physikalisches Institut, Goethe-Universität Frankfurt, Max-von-Laue Stasse 1, \\ 60438 Frankfurt am Main, Germany \\ ${ }^{5}$ Shubnikov Institute of Crystallography, Russian Academy of Sciences, Leninsky Prospekt 59, 119333 Moscow, Russia \\ ${ }^{6}$ Institute of Solid State Physics, Dresden University of Technology, Zellescher Weg 16, D-01062 Dresden, Germany \\ ${ }^{7}$ Saint Petersburg State University, Saint Petersburg 198504, Russia \\ (Received 7 March 2016; revised manuscript received 5 April 2016; published 25 April 2016)
}

\begin{abstract}
The potential of resonant inelastic soft x-ray scattering to measure $4 f$ crystal electric-field excitation spectra in Ce Kondo lattices has been examined. Spectra have been obtained for several Ce systems and show a well-defined structure determined by crystal-field, spin-orbit, and charge-transfer excitations only. The spectral shapes of the excitation spectra can be well understood in the framework of atomic multiplet calculations. For $\mathrm{CeCu}_{2} \mathrm{Si}_{2} \mathrm{we}$ found notable disagreement between the inelastic x-ray-scattering spectra and theoretical calculations when using the crystal-field scheme proposed from inelastic neutron scattering. Modified sets of crystal-field parameters yield better agreement. Our results also show that, with the very recent improvements of soft x-ray spectrometers in resolution to below $30 \mathrm{meV}$ at the $\mathrm{Ce} M_{4,5}$ edges, resonant inelastic x-ray scattering could be an ideal tool to determine the crystal-field scheme in Ce Kondo lattices and other rare-earth compounds.
\end{abstract}

DOI: 10.1103/PhysRevB.93.165134

\section{INTRODUCTION}

Rare-earth (RE) intermetallic compounds attract much attention due to their peculiar electrical, magnetic, and thermal properties. The physics of these systems is mainly determined by the highly correlated electrons in the $4 f$ shell which can hybridize with the wave functions of the surrounding atoms, often resulting in a complex competition between a localized and itinerant nature of the $4 f$ states [1]. This competition is particularly intriguing in $\mathrm{Ce}$ and $\mathrm{Yb}$ systems where the close to one electron or hole occupancy of the $4 f$ shell promotes the formation of Kondo lattices [2]. A precise understanding of $4 f$ based Kondo lattice systems requires knowledge of the crystal electric-field (CEF) scheme, i.e., the splitting of the $4 f$ levels induced by the surrounding charges in the crystal. Recently excited CEF states were even proposed to be at the origin of some of the observed quantum critical points (QCPs) because they induce a metaorbital transition [3-5].

Depending on its symmetry and strength, the CEF potential splits the spin-orbit (SO) multiplets in states of different symmetry and energy. However, determining energy and symmetry of the excited CEF states in a Kondo lattice close to a QCP frequently turned out to be very difficult and unreliable using standard approaches. Such systems have inherently a large Kondo energy scale, which results in broad transitions and thus leads to broad maxima in inelastic neutron scattering (INS), the standard approach to determine the energy of CEF levels. Separating these broad CEF features from the response of the phonons, which are typically in the same energy range, is difficult. In addition, the excitation energy range in INS is

*kurt.kummer@esrf.fr typically limited to 60 to $100 \mathrm{meV}$, which is sometimes not enough to get a complete picture of the CEF scheme [6].

Resonant inelastic x-ray scattering (RIXS) at the rare-earth $M_{4,5}$ edges does not suffer from the phonon problem because of the only indirect coupling to the lattice and has no upper limit in excitation energy. Because of its high sensitivity RIXS does not require large samples of $\mathrm{cm}^{3}$ size, which are often difficult to grow as single crystals. It could therefore offer a new opportunity to address the CEF scheme of RE ions as it has already done for transition-metal compounds, where the CEF splittings are of the order of $1 \mathrm{eV}$ (see, for instance, Ref. [7] and citations therein).

Up to now, the energy resolution achieved in soft $\mathrm{x}$-ray RIXS [8] has not been sufficient to encourage respective RIXS experiments and very few data are available. A new generation of RIXS spectrometers which are being installed or start operation at several synchrotrons worldwide now achieves or will achieve an energy resolution better than $30 \mathrm{meV}$ at the $\mathrm{Ce}$ $M_{4,5}$ edges, which is in most cases sufficient to resolve the CEF splittings and construct the CEF scheme in Ce Kondo lattices. Here, we study the potential of Ce $M_{5}$ RIXS to probe spin-orbit and CEF excitations in several Ce compounds with tetragonal symmetry. Our results show a high sensitivity of RIXS to these $4 f$ excitations, which can be measured essentially free of any background and with no upper limit in the energy of the excitations.

\section{II. $4 \boldsymbol{f}$ EXCITATIONS IN RESONANT INELASTIC X-RAY SCATTERING}

RIXS is a second-order process described by a KramersHeisenberg formula [7]. A core-level electron is resonantly excited into the valence states, leaving an intermediate state 

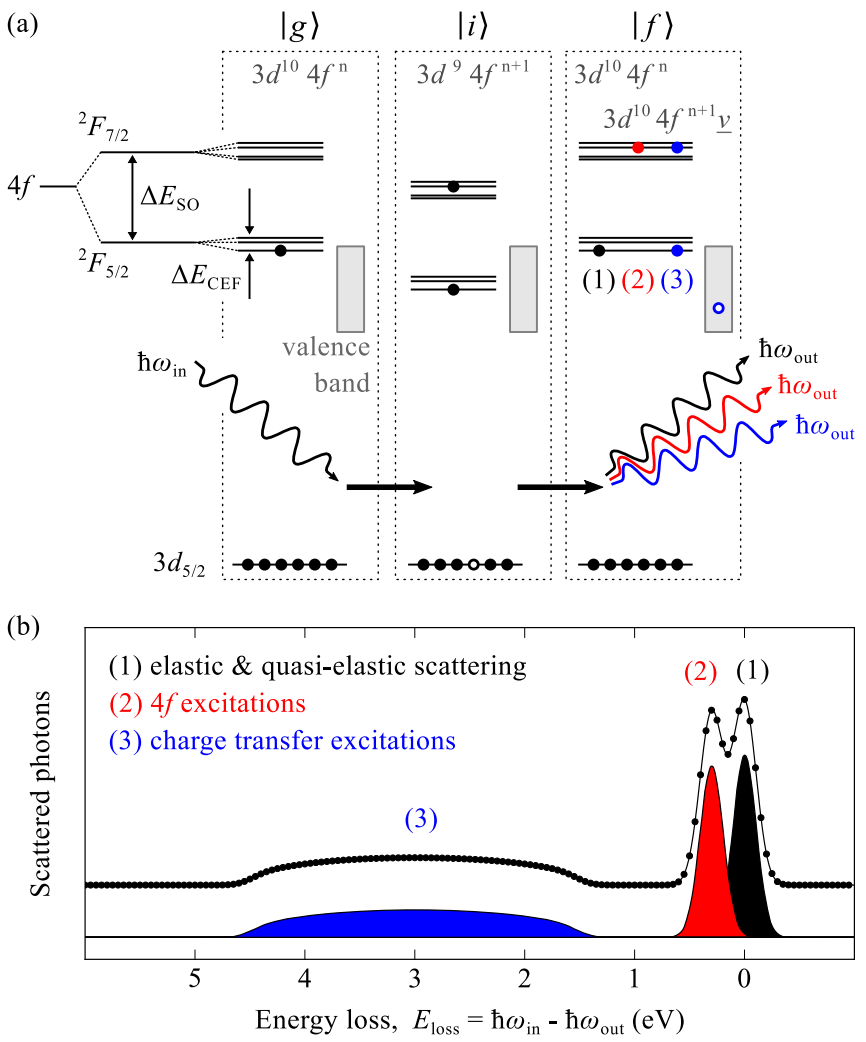

FIG. 1. (a) Scheme of the SO and CEF splitting of the $4 f$ states for a $\mathrm{Ce}^{3+}$ ion in a tetragonal CEF $\left(D_{4 h}\right.$ symmetry) and the dominant elastic and inelastic x-ray-scattering processes at the Ce $M_{5}$ edge. (b) The expected shape of the RIXS spectrum when measured with $\sim 260$-meV resolution.

with a core hole which relaxes into the final state by radiative decay of the core hole. The process is element and orbital specific by tuning the incident photon energy to a specific $\mathrm{x}$-ray-absorption resonance and selecting the appropriate light polarization. A spectrometer is used for measuring the energy and momentum of the scattered photons and thus characterizing the excitations in the material. Because of the high incident photon energy there is no practical upper limit for the energy up to which excitations can be probed.

The $4 f$ excitations in $\mathrm{Ce}$ ions are best probed at $\mathrm{Ce}$ $M_{4,5}$ absorption edges where the dipole selection rules allow direct transition from the Ce $3 d$ to the $4 f$ states and back. Note that although the excitation and de-excitation processes obey the dipole selection rules the entire RIXS process does not. For Ce $3 d \rightarrow 4 f \rightarrow 3 d$ RIXS three processes can be conceived to give a significant spectral contribution, which are schematically shown in Fig. 1(a).

(1) Elastic scattering. The electron excited into the Ce $4 f$ shell falls back into the $3 d$ shell to fill the created core hole. The energies of the incoming and outgoing $x$-ray photons are the same, i.e., no energy has been lost in the sample during the scattering process.

(2) $4 f$ excitations. The core hole in the $3 d$ shell left after excitation of a $3 d$ electron into an unoccupied $4 f$ level is filled by another electron already present in the $4 f$ shell. Comparing the initial and final state this corresponds to promotion of an electron from the $\mathrm{CEF} / \mathrm{SO}$ ground state into an excited state and the energy loss experienced by the scattered photon corresponds to the energy separation between the CEF and SO split levels, which allows us to measure the CEF scheme of the $4 f$ shell. Ce compounds usually possess an intermediate valent ground state with sizable $4 f^{0}$ and $4 f^{1}$ contributions. The $4 f^{2}$ contribution is typically very small because of the strong Coulomb repulsion $U_{f f}$ in the $4 f$ shell. This makes the interpretation of the $4 f$ excitation spectrum very simple. No $4 f$ excitations are expected for the $4 f^{0}$ configuration because for an empty $4 f$ shell in the ground state the intermediate state $3 d$ core hole cannot be filled by another than the excited electron itself. For the $4 f^{1}$ configuration and low enough temperatures only the CEF ground state is populated and all excitations correspond to transitions from this level to higher SO and CEF states. The SO and CEF induced splittings of the $4 f$ levels in Ce ions are shown schematically in Fig. 1(a). SO interaction splits the $4 f$ shell into a threefold degenerate $J=5 / 2$ level and a fourfold degenerate $J=7 / 2$ level. In Ce these two $J$ levels are separated by about $265 \mathrm{meV}$. The presence of a $\mathrm{CEF}$ leads to a further splitting of each of the two $J$ levels in the order of a few tens of meV. The splittings depend both on the symmetry and the strength of the CEF potential. A cubic crystal field lifts the degeneracies within the two $J$ levels only partially, causing a doublet-quartet and a doublet-quartetdoublet structure for the ${ }^{2} F_{5 / 2}$ and ${ }^{2} F_{7 / 2}$ levels, respectively. In a tetragonal CEF the degeneracies are fully lifted, leading to a splitting into three and four Kramers doublets, respectively. Hence in a tetragonal crystal lattice one would expect six $4 f$ excitations to contribute to the RIXS spectrum, in addition to the elastic scattering: two into the two excited ${ }^{2} F_{5 / 2}$ Kramers doublets and four for the ${ }^{2} F_{7 / 2}$ Kramers doublets.

(3) Charge-transfer (CT) excitations. Because of the hybridization of the $4 f$ levels with ligand valence states, i.e., a finite hopping term between the $4 f$ and the valence states, the $3 d$ core hole in the intermediate state can also be relaxed involving a valence electron even though a direct transition would generally be dipole forbidden [9]. This process could be expected to be best seen for the $\mathrm{Ce} 4 f^{0}$ configuration [10]. There hybridization also leads to appearance of a satellite peak in the $M_{5}$ and $M_{4}$ absorption spectrum and not the simple one peak spectrum expected from pure atomic multiplet considerations [11].

These three processes should give rise to a spectral shape of the Ce RIXS spectrum somewhat resembling the schematic spectrum shown in Fig. 1(b). The SO splitting between the ${ }^{2} F_{5 / 2}$ and the ${ }^{2} F_{7 / 2}$ levels of the Ce $4 f^{1}$ configuration is about $265 \mathrm{meV}$. The CEF splittings within the spin-orbit level can amount from a few meV up to many tens of meV. From these figures the Ce RIXS spectrum is expected to have a two component structure, reflecting the ${ }^{2} F_{5 / 2}$ and ${ }^{2} F_{7 / 2}$ spin-orbit levels, with some substructure due to their further splitting by the CEF. The shape and energy of charge-transfer excitations depend on the details of the valence states and their hybridization with the $4 f$ orbitals but they can be expected to be broad and located at higher energies. Typically charge-transfer energies range from one to several $\mathrm{eV}$.

The relative intensity of the three spectral contributions should depend on the specific sample, the scattering geometry, the photon energy, and the incident polarization. The amount 
of elastic scattering will furthermore be somewhat affected by the quality and thus the reflectivity of the surface. It was the aim of our experiment to study the RIXS spectra of several cerium compounds with similar crystal structure and varying CEF in order to understand the potential of RIXS to determine $\mathrm{CEF}$ schemes in cerium and other rare-earth systems.

\section{EXPERIMENTAL DETAILS}

We present here RIXS data taken from three different systems, $\mathrm{Nd}_{2-x} \mathrm{Ce}_{x} \mathrm{CuO}_{4}$ (NCCO) with a $\mathrm{Ce}$ doping level $x=0.16, \mathrm{CeRu}_{2} \mathrm{P}_{2}$, and $\mathrm{CeCu}_{2} \mathrm{Si}_{2}$, where $\mathrm{Ce}$ is in a tetravalent $\left(4 f^{0}\right)$, weakly intermediate valent, and almost trivalent $\left(4 f^{1}\right)$ state, respectively. Single crystals of $\mathrm{CeRu}_{2} \mathrm{P}_{2}$ were obtained as a byproduct in the In-flux crystal growth of CeRuPO [12]. $\mathrm{CeCu}_{2} \mathrm{Si}_{2}$ single crystals were grown using a $\mathrm{Cu}$-flux technique [13]. $\mathrm{CeRu}_{2} \mathrm{P}_{2}$ is a slightly intermediate valent system, with a characteristic energy of the $4 f$ electronic system of the order of $k_{B} T \approx 17 \mathrm{meV}$, as evidenced by maxima in the electrical resistivity $\rho(T)$ [14] and in the magnetic susceptibility $\chi(T)$ [15] at $200 \mathrm{~K} . \mathrm{CeCu}_{2} \mathrm{Si}_{2}$ is a very well known and intensively studied Kondo lattice, being the first discovered heavy fermion superconductor [16]. The NCCO crystals were prepared by a modified flux flow method [17]. All three compounds grow in a tetragonal crystal structure with $D_{4 h}$ symmetry.

The RIXS data were taken at the ESRF beamline ID08 using the AXES spectrometer. The scattering angle $2 \Theta$ was fixed at $130^{\circ}$. The sample surface normal was always along the (001) direction and the spectra were measured in normal emission. All data were taken at low sample temperature, $T<30 \mathrm{~K}$, in order to avoid population of excited crystal-field levels. The combined energy resolution of beamline plus spectrometer was estimated for each measurement using the elastic scattering from an amorphous carbon sample. It amounted to $250 \pm$ 10 meV. X-ray-absorption spectroscopy (XAS) measurements were performed under the same conditions measuring the drain current from the sample.

Simulations were performed with a full multiplet calculation using the SOLID STATE PHYSICS package for Mathematica [18]. The Slater integrals were obtained by atomic HartreeFock values, with scaling factors of $60 \%$ for the $4 f-4 f$ and $80 \%$ for the $3 d-4 f$ Coulomb interaction [19]. The $4 f$ spin orbit splitting was set to $265 \mathrm{meV}$ and the intermediate state broadening was set to $0.5 \mathrm{eV}$.

\section{RESULTS AND DISCUSSION}

We started out by measuring the RIXS spectrum from $\mathrm{NCCO}$ and $\mathrm{CeRu}_{2} \mathrm{P}_{2}$ and comparing with the spectral shapes expected from the discussion above. The almost tetra- and trivalent $4 f$ configurations have been confirmed by comparing the x-ray-absorption spectra with those of $\mathrm{a} \mathrm{CeO}_{2}$ and $\mathrm{a} \mathrm{CeF}_{3}$ reference sample. The XAS data for the Ce $M_{5}$ edge are shown in the left panel of Fig. 2. Because of the strong tendency of $\mathrm{Ce}$ ions towards a mixed valent configuration a pure $\mathrm{Ce}^{4+}$ configuration is rarely observed. Already the spectrum of the $\mathrm{CeO}_{2}$ reference sample deviates from the expected simple two-peak spectral shape. This could be due to contamination of the reference powder sample or stabilization of $\mathrm{Ce}_{3} \mathrm{O}_{4}$ at the surface. In NCCO, Ce serves as electron dopant for the

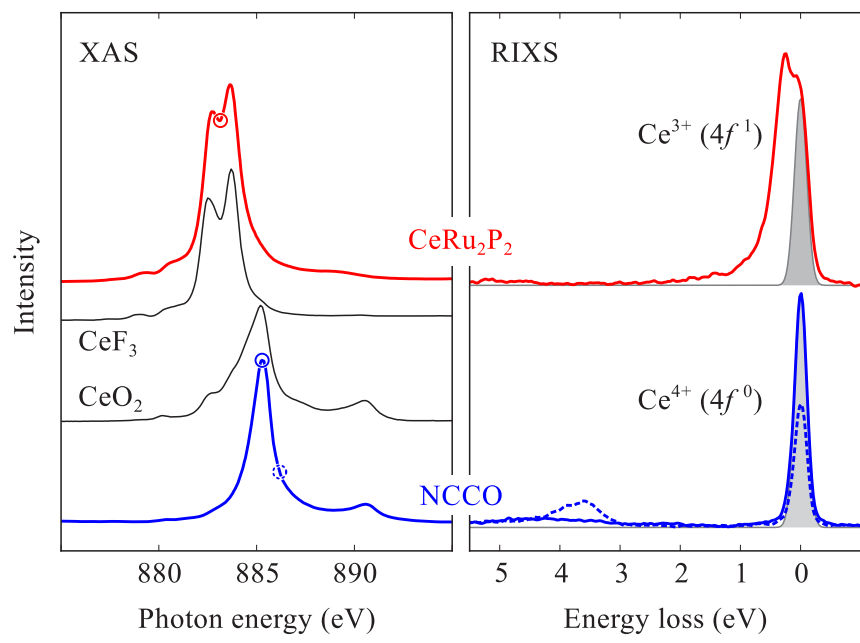

FIG. 2. Left: Ce $M_{5}$ x-ray-absorption spectra of NCCO and $\mathrm{CeRu}_{2} \mathrm{P}_{2}$ together with the $\mathrm{Ce}^{4+}$ and $\mathrm{Ce}^{3+}$ reference spectra taken from a $\mathrm{CeO}_{2}$ and $\mathrm{CeF}_{3}$ powder sample, respectively. Right: RIXS spectra taken from $\mathrm{NCCO}$ and $\mathrm{CeRu}_{2} \mathrm{P}_{2}$. The incident photon energies correspond to those marked with a circle in the absorption spectra. All spectra were measured with $\pi$ polarization of the incident beam.

copper oxygen planes [20] which stabilizes the tetravalent configuration of the Ce ions. The XAS spectrum of $\mathrm{CeRu}_{2} \mathrm{P}_{2}$ strongly resembles that of trivalent $\mathrm{CeF}_{3}$ and thus confirms its only weakly intermediate valent character.

The RIXS spectra of $\mathrm{NCCO}$ and $\mathrm{CeRu}_{2} \mathrm{P}_{2}$ are shown in Fig. 2, right panel. They have been taken with the incident photon energy set to the values marked by circles in the XAS spectra (left panel). With each RIXS spectrum we also show in gray a Gaussian at zero energy and $250-\mathrm{meV}$ full width at half maximum (FWHM), our experimental resolution, in order to give a reference for purely elastic scattering. The $4 f$ RIXS spectrum of NCCO (blue solid line) does not differ from the purely elastic scattering, at least within our experimental resolution, as is expected for $\mathrm{Ce}^{4+}$ from the considerations above. Tuning the incident photon energy towards the CT satellite peak at $\sim 890 \mathrm{eV}$ in the XAS spectrum (blue dashed line) some inelastic intensity appears between 3- and 4-eV energy loss. This intensity corresponds to charge-transfer excitations reflecting the strong hybridization of the $\mathrm{Ce} 4 f$ with the $\mathrm{CuO}_{4}$ valence states.

In contrast, $\mathrm{CeRu}_{2} \mathrm{P}_{2}$ shows a more complex RIXS spectrum (red solid line) with a clear double structure at low energies. The two peaks around 0 and $300 \mathrm{meV}$ energy loss correspond to the ${ }^{2} F_{5 / 2}$ and ${ }^{2} F_{7 / 2}$ spin-orbit levels, respectively. The relative intensity of the inelastic contributions depends a lot on the incident photon energy and is highest in the valley between the two peaks in the XAS. Looking closer at the spectrum there is some low intensity around 1 to $2 \mathrm{eV}$, too high to be due to the CEF splitting. Like in the case of NCCO this intensity can be assigned to CT excitations.

The fact that the CT excitations appear at much lower energy compared to NCCO is consistent with previous results on La compounds [21]. There it was argued that the energy required for CT correlates with the electronegativity of the atoms surrounding the rare-earth ion. This would explain also the results of Watanabe et al. [22], who found lower 


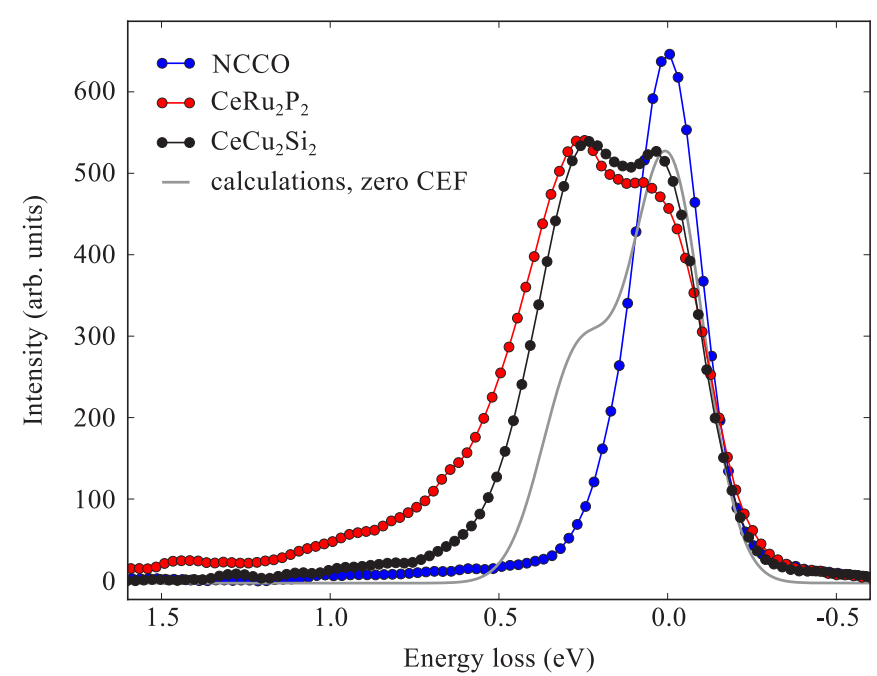

FIG. 3. RIXS spectra of NCCO $\left(\mathrm{Ce}^{4+}\right), \mathrm{CeRu}_{2} \mathrm{P}_{2}\left(\mathrm{Ce}^{3+}\right)$, and $\mathrm{CeCu}_{2} \mathrm{Si}_{2}\left(\mathrm{Ce}^{3+}\right)$ together with calculations of the $\mathrm{Ce}^{3+}$ RIXS spectrum for zero CEF.

energies for CT excitations in $\mathrm{CeRh}_{3}$ compared to $\mathrm{CeO}_{2}$ and calculations by Nakazawa and Kotani [9].

From the data in Fig. 2 it becomes already clear that excitations between different $4 f$ levels are well seen in RIXS and that the experimental data agree well with the expected spectral shapes. It cannot be expected to resolve the CEF splittings in the RIXS spectrum with the experimental resolution of our experiment. This will be reserved for a new generation of RIXS spectrometers with a resolution of the order of the CEF splittings. However, even with the resolution at hand we can detect differences between RIXS spectra taken from systems with different CEF and to spectra calculated for zero crystal field.

This is well seen in Fig. 3, where we compare the RIXS spectrum of $\mathrm{CeRu}_{2} \mathrm{P}_{2}$ with that of $\mathrm{CeCu}_{2} \mathrm{Si}_{2}$. As a reference for elastic scattering, we include the NCCO RIXS spectrum. The $\mathrm{CeCu}_{2} \mathrm{Si}_{2}$ and $\mathrm{CeRu}_{2} \mathrm{P}_{2}$ spectra were taken at the same photon energy marked in Fig. 2 in the XAS of $\mathrm{CeRu}_{2} \mathrm{P}_{2}$. The gray line shows the $\mathrm{Ce}^{3+}$ RIXS spectrum calculated for zero crystal field, i.e., SO interaction only. In this case the ${ }^{2} F_{5 / 2}$ and ${ }^{2} F_{7 / 2}$ levels are each fully degenerate and the resulting RIXS spectrum has only two features. Apparently, this spectrum does not describe the data well, in terms neither of peak positions nor intensity ratios.

There are also clear differences in the $4 f$ excitation spectra of $\mathrm{CeRu}_{2} \mathrm{P}_{2}$ and $\mathrm{CeCu}_{2} \mathrm{Si}_{2}$. Both show a two peak structure separating the ${ }^{2} F_{5 / 2} \rightarrow{ }^{2} F_{5 / 2}$ and the ${ }^{2} F_{5 / 2} \rightarrow{ }^{2} F_{7 / 2}$ excitations but the center of mass of the ${ }^{2} F_{5 / 2} \rightarrow{ }^{2} F_{7 / 2}$ peak in $\mathrm{CeRu}_{2} \mathrm{P}_{2}$ is at notably higher excitation energies. The wider splitting of the $4 f \mathrm{CEF}$ states could be due to more pronounced $\mathrm{CEF}$ effects as a result of the stronger overlap of the $\mathrm{Ru} 4 d$ states with the $\mathrm{Ce} 4 f$ orbitals compared to the $\mathrm{Cu} 3 d$.

In order to resolve the CEF states in the RIXS spectra it is clearly necessary to obtain spectra with roughly an order of magnitude better resolution. However, already from the data at hand we can gain some information on the CEF scheme. We demonstrate this in Fig. 4 with the example of $\mathrm{CeCu}_{2} \mathrm{Si}_{2}$, for which the CEF field has already been studied

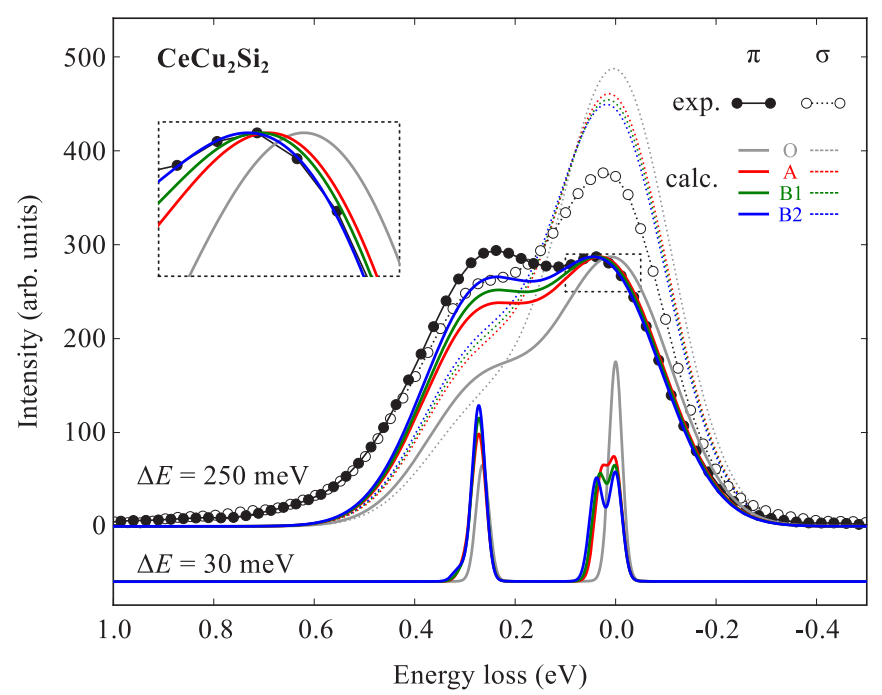

FIG. 4. Experimental RIXS spectra of $\mathrm{CeCu}_{2} \mathrm{Si}_{2}$ taken with $\pi$ and $\sigma$ polarization together with calculated spectra for different $\mathrm{CEF}$ parameters summarized in Table I. The calculated spectra have been broadened with a Gaussian of FWHM $=250 \mathrm{meV}$, the experimental resolution of our experiment, and $30 \mathrm{meV}$, respectively. The inset shows a zoom-in around the low-energy excitations peak.

with INS and magnetic susceptibility as well as specific-heat measurements. We show the experimental RIXS spectra in circles for both $\pi$ (linear horizontal) and $\sigma$ (linear vertical) polarization. The linear dichroism in the RIXS spectra is due to the CEF induced anisotropy of the $4 f$ orbitals. In gray we show again the spectrum obtained in atomic multiplet calculations for zero CEF, with the solid line corresponding to $\pi$ and the dashed line corresponding to $\sigma$ polarization. The red line shows the simulated RIXS spectrum using the CEF parameters obtained from INS [23]. It is in much better agreement with the experimental RIXS spectrum even though it still underestimates the intensity of the ${ }^{2} F_{5 / 2} \rightarrow{ }^{2} F_{7 / 2}$ excitations. The linear dichroism in the calculated RIXS spectrum is much bigger than the experimental result. Looking at the low-energy part of the $\pi$ spectrum in detail one can see that the ${ }^{2} F_{5 / 2} \rightarrow{ }^{2} F_{5 / 2}$ excitations peak is about $10-15 \mathrm{meV}$ lower in energy in the calculated spectrum than in the experimental spectrum (see inset of Fig. 4).

The notable differences in the peak positions as well as the relative intensity of the CEF excitations between the experimental RIXS spectra and simulated spectrum indicate that the CEF scheme proposed in [23] may need to be revised. With the experimental resolution of our experiment and the resulting broad structure of the $4 f$ excitation spectrum it is hard to determine the CEF scheme from our RIXS spectra. However, compared to the INS parameters we obtain an already much better agreement with our experimental data using slightly modified sets of CEF parameters (green and blue curve). The energy positions of the blue curves are in excellent agreement with our experiment, but still do not perfectly describe the intensity ratio between ${ }^{2} F_{5 / 2} \rightarrow{ }^{2} F_{5 / 2}$ and the ${ }^{2} F_{5 / 2} \rightarrow{ }^{2} F_{7 / 2}$ excitations. The corresponding CEF parameters yield an energy of 33 and $41 \mathrm{meV}$ for the lowest 
two excited CEF levels, in contrast to 28 and $29 \mathrm{meV}$ proposed from INS (red curve).

In the calculations, the CEF potential was expressed with a linear combination of renormalized tesseral harmonics, i.e., tesseral harmonics free of any constant prefactor, following Stevens [25]. The coefficients of the expansion, $\hat{A}_{l}^{m}=A_{l}^{m}\left\langle r^{l}\right\rangle$, are free phenomenological parameters to be determined experimentally. We used the $\hat{A}_{l}^{m}$ parameters instead of the historical Stevens parameters $B_{l}^{m}$ for the sake of universality: Stevens's approach is an (extremely good) approximation to calculate the splittings within a multiplet of constant $J$ when $\Delta E_{\mathrm{CEF}} \ll$ $\Delta E_{\mathrm{SO}}$, i.e., when the CEF does not mix the atomic multiplets. But it leads to $J$-dependent crystal-field parameters. In RIXS we are sensitive to both the ${ }^{2} F_{5 / 2}$ and ${ }^{2} F_{7 / 2}$ multiplets and therefore we need to express the CEF potential in a way valid for both $J$. It is always possible to change between the two notations using the relation $B_{l}^{m}=\hat{A}_{l}^{m} \theta(J, l)$, with $\theta$ being a $J$ and $l$-dependent coefficient tabulated by Stevens [25].

For a $\mathrm{Ce}^{3+}$ ion in tetragonal $D_{4 h}$ symmetry, the five parameters $\hat{A}_{2}^{0}, \hat{A}_{4}^{0}, \hat{A}_{4}^{4}, \hat{A}_{6}^{0}$, and $\hat{A}_{6}^{4}$ should be independent and nonvanishing. However, the $\hat{A}_{l}^{m}$ parameters with $l=6$ are usually not considered. This approximation is strictly speaking only valid when considering, with Stevens's approach, excitations within the $J=5 / 2$ multiplet only. This is surely the case in INS, but in practice it has shown its validity experimentally also in the interpretation of Ce $M_{4,5}$ XAS spectra [19,26-29], where both multiplets contribute to the spectrum. With the present resolution, we are insensitive to the fine effects of the $\hat{A}_{6}^{0}$ and $\hat{A}_{6}^{4}$ parameters on the RIXS spectra, which is why we have neglected them here. We list the parameters $\hat{A}_{l}^{m}$ used in the calculations in Table I, together with the corresponding Stevens parameters $B_{l}^{m}$ for the lowest $J=5 / 2$ multiplet.

A critical test of a set of CEF parameters is the comparison of the calculated and measured magnetic susceptibility $\chi(T)$ along main directions of a single crystal. The properties which are the most sensitive to the CEF are the anisotropy and the $T$ dependence of the magnetic susceptibility $\chi(T)$. The anisotropy is very sensitive to the wave functions of the different CEF doublets, especially to those of the CEF ground state. The $T$ dependence is sensitive to the energy split within the $J$ ground-state multiplet (for $\mathrm{Ce} J=5 / 2$ ). For $\mathrm{CeCu}_{2} \mathrm{Si}_{2}$ the anisotropy of $\chi(T)$ and its $T$ dependence has been studied

TABLE I. CEF parameters (in $\mathrm{meV}$ ) used in the calculations shown in Figs. 4 and 5, presented as coefficients of renormalized tesseral harmonics and with the usual Stevens convention for $J=$ $5 / 2$.

\begin{tabular}{lclc}
\hline \hline & $\hat{A}_{2}^{0}$ & \multicolumn{1}{c}{$\hat{A}_{4}^{0}$} & $\left|\hat{A}_{4}^{4}\right|$ \\
& $\mathrm{B}_{2}^{0}$ & \multicolumn{1}{c}{$\mathrm{B}_{4}^{0}$} & $\left|B_{4}^{4}\right|$ \\
\hline O (zero CEF) & 0 & 0 & 0 \\
& 0 & 0 & 0 \\
A (from INS [23,24]) & 22.59 & -0.68 & 71.34 \\
& -1.29 & $-4.3 \times 10^{-3}$ & 0.45 \\
B1 & 19.2 & -1.57 & 94.5 \\
& -1.1 & $-1.0 \times 10^{-2}$ & 0.6 \\
B2 & 19.2 & -2.3 & 118 \\
& -1.1 & $-1.5 \times 10^{-2}$ & 0.75 \\
\hline \hline
\end{tabular}

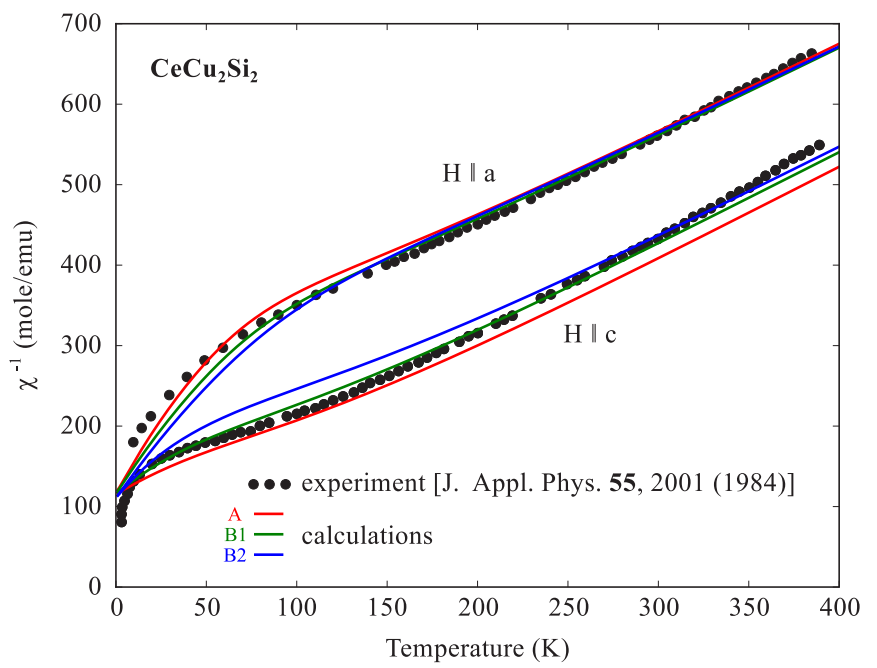

FIG. 5. Magnetic susceptibility of $\mathrm{CeCu}_{2} \mathrm{Si}_{2}$ along the two principal crystallographic directions together with calculated curves for different CEF parameters summarized in Table I and using a molecular-field constant of $110 \mathrm{~mole} / \mathrm{emu}$.

by different groups, with quite similar results. In Fig. 5 we plot the experimental data $\chi^{-1}(T)$ obtained by Batlogg et al. [30] for a field applied along the $c$ direction and the basal plane of $\mathrm{CeCu}_{2} \mathrm{Si}_{2}$. We computed the susceptibility expected for the different CEF parameter sets in Table I using the SOLID STATE PHYSICS code [18]. The parameters tuned to fit the RIXS results reproduce the susceptibility of $\mathrm{CeCu}_{2} \mathrm{Si}_{2}$ along both directions at least as well as the original parameter of the INS study and thus fulfill an essential criterion for being meaningful.

However, the agreement with the experimental RIXS spectra is still not perfect and the broad structures of our experimental spectrum do not give high enough sensitivity to changes in the CEF parameters to unambiguously pin down the CEF levels. An improvement by a factor of 10 in experimental resolution would be needed to resolve the CEF splittings. At the bottom of Fig. 4 we show the simulated spectra assuming a resolution of $30 \mathrm{meV}$ instead of $250 \mathrm{meV}$. This resolution has been achieved recently with the first of a new generation of soft x-ray RIXS spectrometers and will become available at other synchrotron facilities over the next few years, too. At this experimental resolution a proper experimental characterization of the CEF scheme in rare earth becomes possible. One could also already expect to see a broadening of some of the CEF excitations beyond the elastic linewidth due to the large Kondo scale in many Ce Kondo lattices. Such broadenings would be a direct consequence of the dispersion of the $4 f$ CEF levels observed in $\mathbf{k}$-resolved single-particle excitation spectra obtained by means of angleresolved photoemission spectroscopy [31].

\section{SUMMARY}

We have demonstrated the capabilities of soft x-ray RIXS for studying CEF and spin-orbit excitations in Ce Kondo lattices. Due to the weak coupling of the photons to the crystal lattice RIXS does not suffer from the "phonon problem" of INS. The spectra are well defined with strong $4 f$ excitations 
between 0 and $400 \mathrm{meV}$ and weaker charge-transfer excitations at higher energies. All spectral features are well understood within atomic multiplet calculations. For the Kondo lattice $\mathrm{CeCu}_{2} \mathrm{Si}_{2}$ we have seen a discrepancy between the CEF scheme determined from INS measurements and our experimental data. A modified set of CEF parameters is able to better describe our results. Improvements in the experimental resolution to $30 \mathrm{meV}$ or below, which have been demonstrated very recently for the $\mathrm{Cu} L_{3}$ edge [32], will allow us to unambiguously determine CEF schemes in Ce Kondo lattices from soft X-ray RIXS measurements. RIXS could also be used to determine the CEF in other rare-earth compounds where INS experiments are difficult because of the high absorption cross section of the rare-earth ions or small sample sizes.

\section{ACKNOWLEDGMENT}

D. V. Vyalikh acknowledges Saint Petersburg State University for Research Grant No. 15.61.202.2015.
[1] A. Mackintosh, in New Trends in Magnetism, Magnetic Materials, and their Applications (Springer, New York, 1994), pp. 387-391.

[2] B. Coqblin, M. Gusmão, J. Iglesias, C. Lacroix, S. Magalhães, A. Ruppenthal, A. Schmidt, and A. Theumann, in Electron Correlations and Materials Properties 2 (Springer, New York, 2003), pp. 159-179.

[3] L. V. Pourovskii, P. Hansmann, M. Ferrero, and A. Georges, Phys. Rev. Lett. 112, 106407 (2014).

[4] J.-P. Rueff, J. M. Ablett, F. Strigari, M. Deppe, M. W. Haverkort, L. H. Tjeng, and A. Severing, Phys. Rev. B 91, 201108 (2015).

[5] E. Lengyel, M. Nicklas, N. Caroca-Canales, and C. Geibel, Phys. Rev. B 88, 155137 (2013).

[6] S. Patil, K. Kummer, A. Hannaske, C. Krellner, M. Kuhnt, S. Danzenbächer, C. Laubschat, C. Geibel, and D. V. Vyalikh, JPS Conf. Proc. 3, 011001 (2014).

[7] L. J. P. Ament, M. van Veenendaal, T. P. Devereaux, J. P. Hill, and J. van den Brink, Rev. Mod. Phys. 83, 705 (2011).

[8] G. Ghiringhelli, A. Piazzalunga, C. Dallera, G. Trezzi, L. Braicovich, T. Schmitt, V. N. Strocov, R. Betemps, L. Patthey, X. Wang, and M. Grioni, Rev. Sci. Instrum. 77, 113108 (2006).

[9] M. Nakazawa and A. Kotani, J. Phys. Soc. Jpn. 71, 2804 (2002).

[10] M. Nakazawa, H. Ogasawara, and A. Kotani, J. Phys. Soc. Jpn. 69, 4071 (2000).

[11] A. Kotani and H. Ogasawara, J. Electron Spectrosc. Relat. Phenom. 60, 257 (1992).

[12] C. Krellner and C. Geibel, J. Cryst. Growth 310, 1875 (2008).

[13] S. Seiro, M. Deppe, H. Jeevan, U. Burkhardt, and C. Geibel, Phys. Status Solidi B 247, 614 (2010).

[14] T. Fujiwara, K. Kanto, K. Matsubayashi, Y. Uwatoko, and T. Shigeoka, Journal of Physics: Conference Series 273, 012112 (2011).

[15] C. Krellner (private communication).

[16] F. Steglich, J. Aarts, C. D. Bredl, W. Lieke, D. Meschede, W. Franz, and H. Schäfer, Phys. Rev. Lett. 43, 1892 (1979).

[17] I. Makarova, A. Bram, H. Burzlaff, M. Blomberg, M. Merisalo, and V. Simonov, J. Alloys Compd. 225, 599 (1995).

[18] M. W. Haverkort, M. Zwierzycki, and O. K. Andersen, Phys. Rev. B 85, 165113 (2012).

[19] T. Willers, D. T. Adroja, B. D. Rainford, Z. Hu, N. Hollmann, P. O. Körner, Y.-Y. Chin, D. Schmitz, H. H. Hsieh, H.-J. Lin, C. T. Chen, E. D. Bauer, J. L. Sarrao, K. J. McClellan, D. Byler,
C. Geibel, F. Steglich, H. Aoki, P. Lejay, A. Tanaka, L. H. Tjeng, and A. Severing, Phys. Rev. B 85, 035117 (2012).

[20] K. Ishii, M. Fujita, T. Sasaki, M. Minola, G. Dellea, C. Mazzoli, K. Kummer, G. Ghiringhelli, L. Braicovich, T. Tohyama, K. Tsutsumi, K. Sato, R. Kajimoto, K. Ikeuchi, K. Yamada, M. Yoshida, M. Kurooka, and J. Mizuki, Nat. Commun. 5, 3714 (2014).

[21] C. Dallera, K. Giarda, G. Ghiringhelli, A. Tagliaferri, L. Braicovich, and N. B. Brookes, Phys. Rev. B 64, 153104 (2001).

[22] M. Watanabe, Y. Harada, M. Nakazawa, Y. Ishiwata, R. Eguchi, T. Takeuchi, A. Kotani, and S. Shin, Surf. Rev. Lett. 09, 983 (2002).

[23] E. A. Goremychkin and R. Osborn, Phys. Rev. B 47, 14280 (1993).

[24] E. Goremychkin, A. Y. Muzychka, and R. Osborn, J. Exp. Theor. Phys. 83, 738 (1996).

[25] K. W. H. Stevens, Proc. Phys. Soc. London, Sect. A 65, 209 (1952).

[26] P. Hansmann, A. Severing, Z. Hu, M. W. Haverkort, C. F. Chang, S. Klein, A. Tanaka, H. H. Hsieh, H.-J. Lin, C. T. Chen, B. Fåk, P. Lejay, and L. H. Tjeng, Phys. Rev. Lett. 100, 066405 (2008).

[27] T. Willers, Z. Hu, N. Hollmann, P. O. Körner, J. Gegner, T. Burnus, H. Fujiwara, A. Tanaka, D. Schmitz, H. H. Hsieh, H.-J. Lin, C. T. Chen, E. D. Bauer, J. L. Sarrao, E. Goremychkin, M. Koza, L. H. Tjeng, and A. Severing, Phys. Rev. B 81, 195114 (2010).

[28] T. Willers, B. Fåk, N. Hollmann, P. O. Körner, Z. Hu, A. Tanaka, D. Schmitz, M. Enderle, G. Lapertot, L. H. Tjeng, and A. Severing, Phys. Rev. B 80, 115106 (2009).

[29] F. Strigari, T. Willers, Y. Muro, K. Yutani, T. Takabatake, Z. Hu, Y.-Y. Chin, S. Agrestini, H.-J. Lin, C. T. Chen, A. Tanaka, M. W. Haverkort, L. H. Tjeng, and A. Severing, Phys. Rev. B 86, 081105 (2012).

[30] B. Batlogg, J. P. Remeika, A. S. Cooper, and Z. Fisk, J. Appl. Phys. 55, 2001 (1984).

[31] D. V. Vyalikh, S. Danzenbächer, Y. Kucherenko, K. Kummer, C. Krellner, C. Geibel, M. G. Holder, T. K. Kim, C. Laubschat, M. Shi, L. Patthey, R. Follath, and S. L. Molodtsov, Phys. Rev. Lett. 105, 237601 (2010).

[32] N. B. Brookes, plenary talk delivered at the Synchrotron Radiation and Instrumentation Conference 2015, New York, 2015. 